\title{
Highlights of the ISOLDE facility and the HIE-ISOLDE Project
}

\author{
M.J.G BorGe
}

PH Department, CERN, CH-1211 Geneva-23, Switzerland Instituto de Estructura de la Materia, CSIC, Serrano 113bis, 28006-Madrid, Spain

\begin{abstract}
The ISOLDE Radioactive Beam Facility is the dedicated CERN installation for the production and acceleration of radioactive nuclei far from stability. Exotic nuclei of most chemical elements are available for the study of nuclear structure, nuclear astrophysics, fundamental symmetries and atomic physics, as well as for applications in condensed-matter and life sciences. Since more than a decade it offers the largest variety of post-accelerated radioactive beams in the world today. In order to broaden the scientific opportunities beyond the reach of the present facility, the on-going HIE-ISOLDE (High Intensity and Energy) project will provide major improvements in energy range, beam intensity and beam quality. Post-accelerated beams will be available already this year boosting the beam energy of the current REX LINAC to $4.3 \mathrm{MeV} / \mathrm{u}$ reaching $5.5 \mathrm{MeV} / \mathrm{u}$ next spring. In this new energy regime the Coulomb excitation cross sections are strongly increased with respect to the previous energy of bearly $3 \mathrm{MeV} / \mathrm{u}$ and many transfer reaction channels will open. The second stage of the energy upgrade will allow energies of the beam up to $10 \mathrm{MeV} / \mathrm{u}$ for the worst scenario of $\mathrm{A} / \mathrm{q}=4.5$. The funds are already secured and it is expected to be completed in 2017. In this contribution the present status of the ISOLDE facility will be discussed, some highlights will be briefly described to illustrate the advances of the facility. The HIEISOLDE project will be described together with a panorama of the physics cases to be addressed in the near future with emphasis in the day-one experiment to be done this year.
\end{abstract}




\section{The ISOLDE facility}

The ISOLDE Radioactive Beam Facility is the CERN experiment for the production and acceleration of radioactive nuclei. Isotopes from a variety of chemical elements are produced in a target directly connected to the ion-source of an isotope separator to minimise the time delay between production of a nucleus and its arrival at the experimental setup.

This ISOL-type facility which started in 1964 at the $600 \mathrm{MeV}$ proton Synchrocyclotron is located since 1992 at the Proton-Synchrotron Booster (PSB) of the European Organization for Nuclear Research, CERN. Pulses of about $310^{13}$ protons are sent to the ISOLDE target every 1.2 seconds or multiples of this period resulting in an average beam intensity of 2 particle $\mu \mathrm{A}$ [1]. The use of high-energy protons such as the ones delivered by the CERN PSB injector of $1.4 \mathrm{GeV}$ has been recognized to be optimum for the production of radioactive nuclei via spallation, fission and fragmentation reactions on thick targets. As many as twenty different solid and liquid target materials are used to produce a wide spectrum of radioactive isotopes covering essentially the whole nuclear chart below uranium $(Z=92)$. The reaction products are thermalized in the bulk of the target material, and thereafter transported from the target container to an ion source via diffusion and effusion processes. The speed of the diffusion and effusion process is governed by the physical and chemical properties of the radioactive elements of interest and of the specific materials that the target container, transfer line and ion source are made of. Chemical selectivity is obtained by the right combination of target-ion sources giving rise to a selective production of more than 1300 isotopes of 73 different chemical elements, being Boron the last element incorporated this year to this long list. ISOLDE's decades of experience in target-ion source development makes the whole process, from isotope production in target to beam delivery to experiments, efficient, fast and selective giving rise to a wide spectrum of beams. Fig. 1 overlays on the nuclear chart the ISOLDE beams with their production yields. The list is not fully updated. Radioactive beams has been produced with intensities reaching the $\mathrm{nA}$ level (e.g. ${ }^{213} \mathrm{Fr}$ with $8.410^{9}$ particles per second) and of very short-lived nuclei with a few milliseconds half life (e.g. $\left.{ }^{14} \mathrm{Be}, T_{1 / 2}=4.45 \mathrm{~ms}\right)$. These beams are used for experiments in the field of nuclear physics (taking $\sim 67 \%$ of the beam time), weak interaction studies ( $\sim 3 \%)$, solid-state physics studies $(\sim 19 \%)$, nuclear astrophysics $(\sim 4 \%)$ and, biology and medicine ( 8 \%). Statistics given for 2014 .

The ions are subsequently extracted from the ion source, accelerated to $60 \mathrm{keV}$, mass separated according to mass-over-charge ratio using a dipole 


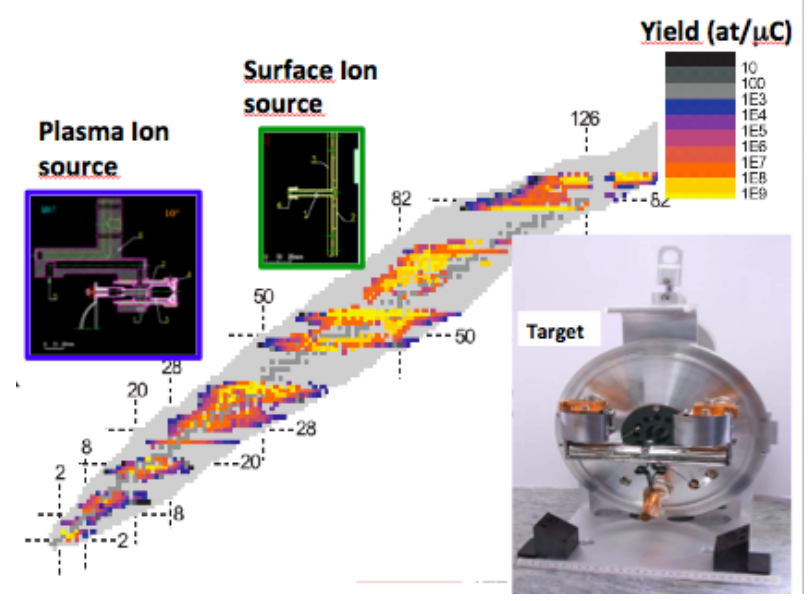

Figure 1: Chart of nuclide colour code follows the ISOLDE yields, scale given in the upper right corner. The gaps with no beams available correspond to refractory-type elements. Off diagonal to the left the surface and plasma ion source designs are shown, and to the right a photo of the target unit.

magnet and send to the different experimental set-ups. Two mass separator units exit with different mass resolution power, the GPS with $\mathrm{M} / \delta \mathrm{M}=$ 1000 and the HRS with $\mathrm{M} / \delta \mathrm{M}=5000$. A radiofrequency cooler-buncher was installed in 2006 after the HRS allowing to deliver short bunches of radioactive ion beams to experiments without a substantial loss in intensity. This development has resulted in an important increase in the peak-tobackground ratio for some experiments e.g. the collinear laser spectroscopy measurements [2], and a even better beam waist for all of them. A general description of the ISOLDE facility is given in [1].

The success of ISOLDE is due to the continuous development of new radioactive ion beams and to the improvement of the experimental conditions. Decades of RIB research has shown that different purification steps have to be applied one after the other as in most cases none of the purification steps result in a $100 \%$ pure beams. Different ways are implemented to purify ISOLDE's beams. The most straightforward way is increasing the wanted isotope over its isobaric contaminants using the high-resolution mass separator [1]. Using the high-resolution mass separator, however, often induces losses in intensity. Other ways to purify the beam is making use of the physical and chemical properties of the element. The most elegant and effective way to purify the ion beam is the use of the so-called 'Resonant Ionisation Laser Ion Source (RILIS)' [3]. Resonant laser ionisation is an 
element selective process where atoms are irradiated by two or three lasers exciting the electron in two or three resonant steps into the continuum. This allows the production of very pure beams as only atoms of the element of interest are ionized.

RILIS has become extensibly used at ISOLDE [4] because of its ability to efficiently ionize a range of 35 elements with an unmatched degree of element selectivity. In recent years, the RILIS team has contributed to a highly successful collaboration with several experimental groups at ISOLDE [5]. This collaboration has performed extensive laser and nuclear-spectroscopic studies of shape evolution/coexistence in the lead region using the technique of in-source laser spectroscopy. This is the most sensitive laser spectroscopy method available at ISOL facilities, and has enabled the measurement of isotopes with a yield as low as 0.1 ions/second. In particular, long chains of $\mathrm{Pb}$ $(\mathrm{Z}=82)[6,7], \mathrm{Po}(\mathrm{Z}=84)[8,9], \mathrm{Tl}(\mathrm{Z}=81)$ [10-12], isotopes have been studied. This year At $(Z=85), \mathrm{Au}(\mathrm{Z}=79)$ and $\mathrm{Hg}(\mathrm{Z}=80)$ have been studied employing a combination of particle detection techniques: $(\alpha / \beta / \gamma)$ with the Windmill setup from Leuven (Belgium); direct ion counting with the ISOLTRAP multi-reflection time of flight mass spectrometer (MR-TOF MS); and ion beam current measurements using the ISOLDE faraday cups. The combination of all these methods has allowed to determine the hyperfine structure measured by RILIS of the neutron defficient $\mathrm{Hg}$ isotopes from $\mathrm{A}=177$ to 181 . The mean square radius shows absence of the odd-even staggering observed in the ${ }^{181-184} \mathrm{Hg}$ isotopes. This confirms the predictions than well below the mid-shell neutron number $\mathrm{N}=104$ the structure of the $\mathrm{Hg}$-isotopes becomes spherical [13]. Using the same techniques the radii of the n-defficient $\mathrm{Au}$ isotopes from $\mathrm{A}=176-183$ were measured. The results show that the deformed $\mathrm{Au}$ isotopes are symmetric around the mid-shell $\mathrm{N}=104$ and return to sphericity for neutron defficient isotopes lighter than ${ }^{179} \mathrm{Au}$.

In order to boost the energy of the existing radioactive ion beams of ISOLDE from typically $60 \mathrm{keV}$ to a few $\mathrm{MeV} / \mathrm{u}$ a new concept was proposed in which the existing singly-charged ion beams from ISOLDE were accelerated in a universal, fast, efficient and cost-effective way $[14,15]$. The concept was based on ion beam cooling and bunching in the buffer gas of a Penning trap [16], charge-state breeding in an Electron Beam Ion Source (EBIS) [17] and post-acceleration in a room-temperature linear accelerator [18]. The $60 \mathrm{keV}$ radioactive ion beams from ISOLDE are retarded and continuously injected into a Penning trap situated at a high voltage platform. In the trap the ions are accumulated and at the same time cooled via buffer gas collisions. After a fixed time, the ion bunch is extracted from the Penning trap and transferred to the EBIS. The typical duration of the ion 


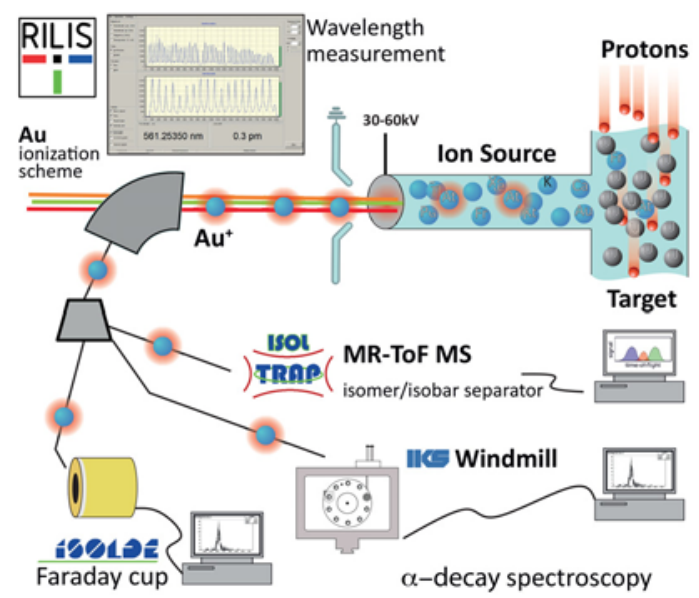

Figure 2: Picture of the RILIS ion source selection system combine with the different devices used used for $\alpha / \beta / \gamma$ detection by the Leuven windmill device, ion selection and counting using the ISOLTRAP MR-ToF mass spectrometer or ion beam current in the ISOLDE Faraday cup.

bunches extracted from the Penning trap is $10 \mu \mathrm{s}$. After charge breeding to the required mass-to-charge ratio $(\mathrm{A} / \mathrm{q})$ between $2.5-4.5$, the ion bunch is extracted from the EBIS, mass separated and injected in the radiofrequency quadrupole (RFQ) accelerator. The charge breeding time depends on the required charge-to-mass ratio and thus on the isotope of interest. It varies from typically $20 \mathrm{~ms}$ for light mass elements $(\mathrm{A}<40)$ to over $200 \mathrm{~ms}$ for the heavy elements in the lead region $(\mathrm{A} \simeq 200)$. The room temperature accelerator is typically running with a $10 \%$ duty cycle. The RFQ accelerator is followed by an interdigital H-type (IH) structure with a final energy between 1.1 and $1.2 \mathrm{MeV} / \mathrm{u}$, three seven-gap resonators that create an energy range from 0.8 to $2.2 \mathrm{MeV} / \mathrm{u}$ and finally by a nine-gap resonator boosting the beam energy to a maximum of about $3 \mathrm{MeV} / \mathrm{u}$.

While the original goals of the REX-ISOLDE project were limited to energies up to $2 \mathrm{MeV} / \mathrm{u}$ and masses below $A=50$, the universal concept proved to be very successful and meanwhile beams with $\mathrm{A} / \mathrm{q}$ ratio $<4.5$ and with masses up to 224 have been accelerated up to $3 \mathrm{MeV} / \mathrm{u}$, with efficiencies with varying from $\sim 2$ to $10 \%$. The experimental program with post-accelerated beams focussed on Coulomb excitation, few-nucleon transfer reaction studies and fusion evaporation studies using radioactive beams. Dedicated instrumentation for $\gamma$-ray and charge particle detection was developed especially tailored to the particularities of radioactive ion beam research: low beam 
intensities, low- $\gamma$-ray multiplicity and strong background radiation from the radioactivity of the beam itself. For $\gamma$-ray detection, an array of six-fold segmented, individually encapsulated and tapered germanium crystals was constructed [19]. The so-called Miniball array is optimized with respect to full-energy peak efficiency and angular resolution rather then resolving power.

Miniball consists of eight cryostats, each containing three individual germanium crystals, that are mounted in close geometry around the target position. In its standard configuration for Coulomb excitation experiments, the typical target to detector distance is about $11 \mathrm{~cm}$ resulting in a solidangle coverage of $\sim 60 \%$ of $4 \pi$. The photo-peak efficiency for the $1.3 \mathrm{MeV}$ reaches up to about $8 \%$. In case of Coulomb excitation experiments the spherical detection chamber is equipped with a double sided silicon strip detector (DSSSD) of the CD type [20]. The DSSSD covers an angular range typically from $16^{\circ}$ to $53^{\circ}$ laboratory angle in the forward direction. This range can be changed by changing the target to detector distance. To study few-nucleon transfer reactions using radioactive ion beams in inverse kinematics, two detection set-ups were developed. The first one mainly used for transfer reactions in the light mass range consisted out of one or more [21] Si double-sided strip detectors with a specially developed [22] thin dead layer. The second set-up, called T-REX (the T stands for transfer), is mainly used for transfer reactions using heavier mass beams and combines particle detection with gamma detection $[23,24]$. It consists of the Miniball array to detect $\gamma$-rays in coincidence with particles detected by an array of segmented $\mathrm{Si}$ detectors. The array comprises two so-called CD detectors, in forward and backward direction, and a barrel of eight planar DSSSD's around $90^{\circ}$. A good description of the REX-ISOLDE experiments done during its first decade of existence can be found in [25]

The heaviest accelerated REX-ISOLDE beams of Radon and Radium nuclei were employed to investigate shape asymmetric configurations. Strong octupole correlations leading to pear shapes can arise when nucleons near the Fermi surface occuping states of opposite parity with orbital and total angular momentum differing by 3 units. Pear shape nuclei have enhanced E1 and E3 transitions connecting rotational states of opposite parity. The E1 moments are small and dominated by single-particle and cancellation effects, while the E3 transition moments are collective in behaviour and insensitive to single-particle behaviour. Octupole correlations were studied by the determination of the electric octupole transition strengths in ${ }^{220} \mathrm{Rn}$ and ${ }^{224} \mathrm{Ra}$. The E3 moments are an observable that should provide direct evidence for enhanced octupole correlations for deformed nuclei. The measured E1, E2 


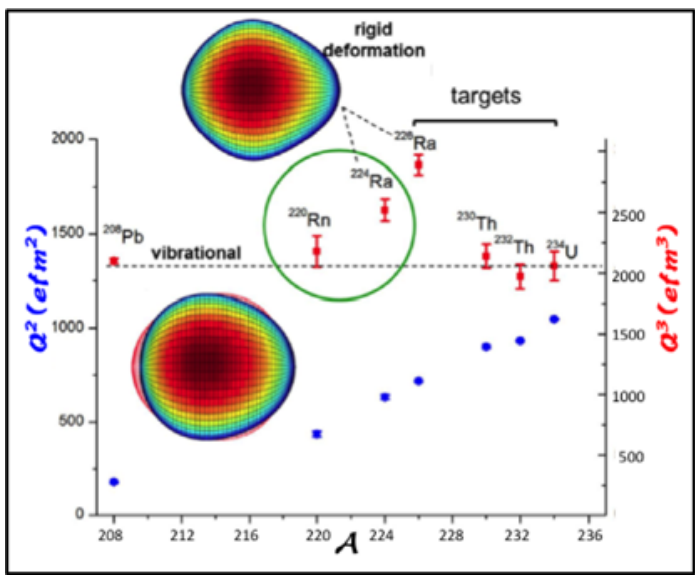

Figure 3: Systematics of the quadrupole and octupole moments for heavy nuclei with $\mathrm{A}=208-234$ including the new cases measured at ISOLDE ${ }^{220} \mathrm{Rn}$ and ${ }^{224} \mathrm{Ra}$. The present results indicate a clear evidence for strong octupole deformation in ${ }^{224} \mathrm{Ra}[26]$.

or E3 transitions for ${ }^{220} \mathrm{Rn}$ and ${ }^{224} \mathrm{Ra}$ allowed the determination of the reduced matrix elements and the intrinsic moments. As shown in Fig. 3, the E2 moment increases a factor of 6 from ${ }^{208} \mathrm{~Pb}$ to ${ }^{334} \mathrm{U}$ while the E3 moment only varies up to $50 \%$ in the same region. The large values of Q3 measured for ${ }^{220} \mathrm{Rn}$ and ${ }^{224} \mathrm{Ra}$ point to an enhancement of octupole collectivity indicating the onset of octupole deformation in this region. While ${ }^{220} \mathrm{Ra}$ has Q3 values typical of an octupole vibrator, the Q3 values obtained for ${ }^{224} \mathrm{Ra}$ gives convincing evidence that this nucleus is of quadrupole-octupole shape in its ground state. These relevant results for nuclear structure and of importance for EDM searchers are summarized in a recent Nature paper [26].

\section{The HIE-ISOLDE project}

The HIE-ISOLDE upgrade (HIE stands for High Intensity and Energy), intends to improve the experimental capabilities at ISOLDE over a wide front [27]. The main features are to boost the energy of the beams, going in steps from currently $3 \mathrm{MeV}$ via $5.5 \mathrm{MeV}$ to finally $10 \mathrm{MeV}$ per nucleon, and to increase roughly sixfold the production due to an increase in intensity and energy of the proton injector. In addition improvements in several aspects of the secondary beam properties such as purity, ionisation efficiency and optical quality are addressed in the project, see Fig. 4.

Major project components include a new superconducting (SC) linear ac- 


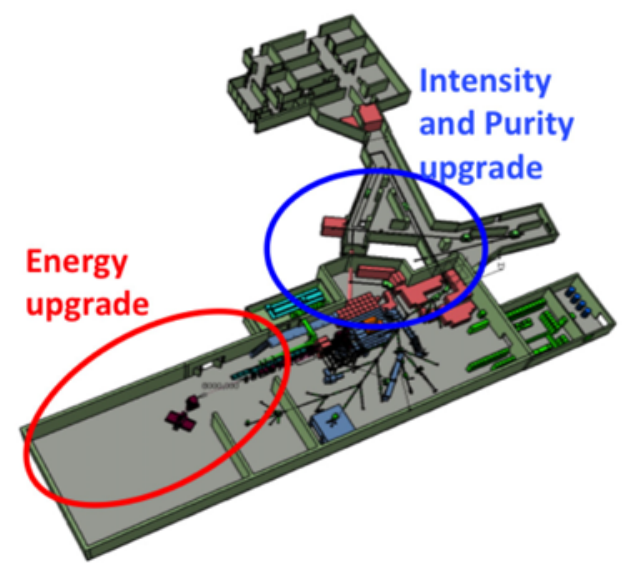

Figure 4: Layout of the ISOLDE facility showing the target-ion source unit connected to each mass separator as well as the the post accelerator line at the experimental hall. The expected upgrades of the facility are indicated: Increase in intensity and energy of the injector in 2020; increase of the post-accelerator beam from $4.3 \mathrm{MeV} / \mathrm{u}$ this year to $10 \mathrm{MeV} / \mathrm{u}$ in 2017 . Improvements of the beam purity and quality are a continuos effort of the technical team.

celerator (LINAC) based on Quarter Wave resonators (QWRs) for the postacceleration and the necessary $4.5 \mathrm{~K}$ cryogenic station for helium. The decision to keep the existing experimental hall has imposed severe constraints on the LINAC, so it has been necessary to design and build accelerating cavities with a very high voltage gradient of $6 \mathrm{MV} / \mathrm{m}$ and low heat dissipation below $10 \mathrm{~W}$. The superconducting accelerator part dedicated to the increase of energy is based on a quart wave resonator (QWR) geometry with twenty high- $\beta$ cavities cooled by helium and installed in four cryo-modules. The transverse focusing is achieved using four superconducting solenoids housed inside the cryo-modules maximising the transverse acceptance. Each cryomodule contains five cavities based on 101.28 MHz niobium-sputtered copper $(\mathrm{Nb} / \mathrm{Cu})$ Quarter Wave Resonator and one solenoid, see inset of Fig. 5. The first two cryo-modules with five high- $\beta$ cavities each will permit to increase the energy to $5.5 \mathrm{MeV} / \mathrm{u}$ for $\mathrm{A} / \mathrm{q}=4.5$ and constitute the first phase of the project. The first cryo-module was installed at ISOLDE in May. Hardware commissioning of the first cryo-module of the HIE-ISOLDE superconducting LINAC has been now ongoing for the last 3 months, with a start planned for mid-October 2015. Two beam lines are already operative for physics and a third beam line will be built next year.

The first call for proposals was made in October 2012. So far thirty 
four experiments have been approved with more than six hundred $8 \mathrm{~h}$ shifts allocated. The physics cases approved expand over the wide range of postaccelerated beams available at ISOLDE, where the increase in energy of the radioactive beams will enhance the cross section in most of the cases and the accessibility to detail nuclear structure information at higher excitation energy.

In the light nuclear region, reaction studies of astrophysical interest such as the search for high-excited states in ${ }^{8} \mathrm{Be}$ to address the cosmological ${ }^{7} \mathrm{Li}$ problem. Nuclear structure studies are planned to characterise cluster structure in ${ }^{10} \mathrm{Be}$ by transfer reaction. Characterisation of resonant states in the proton-rich nucleus ${ }^{21} \mathrm{Al}$ will be determined by resonance elastic and inelastic scattering using the active target MAYA in order to check isospin conservation beyond the drip line. For middle mass nuclei, the validity of a shell model description around ${ }^{78} \mathrm{Ni}$ will be studied as well as shape coexistence in the region $\mathrm{A}=70-80$ will be determined with high precision. Statistical properties of warm nuclei will be investigated by the low-energy enhancement of the gamma strength function of neutron rich nuclei. For heavier mass nuclei, quadrupole and octupole collectivity will be addressed in the neutron rich $\mathrm{Te}$, Xe and $\mathrm{Ba}$ isotopes by Coulomb excitation, lifetime measurements and magnetic moment determination. Collective effects around the double magic ${ }^{132} \mathrm{Sn}$ will also be studied. For heavy nuclei, shape coexistence in the light $\mathrm{Pb}$ isotopes will be explored. Measurements of octupole collectivity in the Rn and Ra nuclei using Coulomb excitation will continue. In the quest of super-heavies, it is proposed to investigate the influence of the predicted shell closures at $\mathrm{Z}=120$ and $\mathrm{N}=184$ by probing the height of the fission barrier in the compound nucleus. This will be achieved by exploring the contributions of quasi-fission and fusion-fission reactions; in particular by the use of the reaction of the deformed ${ }^{95} \mathrm{Rb}$ beam on a ${ }^{209} \mathrm{Bi}$ target is expected to permit the study of these features.

The proposed studies will be realised with the existing workhorses Miniball [19] and T-REX [28] plus new instrumentation for transfer reaction studies such as the active targets MAYA [29] and the future ACTAR, a new general purpose scattering chamber, the two arms CORSET setup from GSI,...etc. The Miniball array will be complemented with an electron spectrometer, SPEDE, to realise Coulomb excitation studies of odd-heavy nuclei. While writing these pages the first beams of carbon and rubidium have been accelerated preparing the machine to start with radioactive $\mathrm{Zn}$ beam for the 10th of October 2015. The nuclei approved for physics experiments at HIEISOLDE are shown in red in the chart of the nuclides displayed in Fig. 5. We will start with the study of the evolution of the nuclear structure 


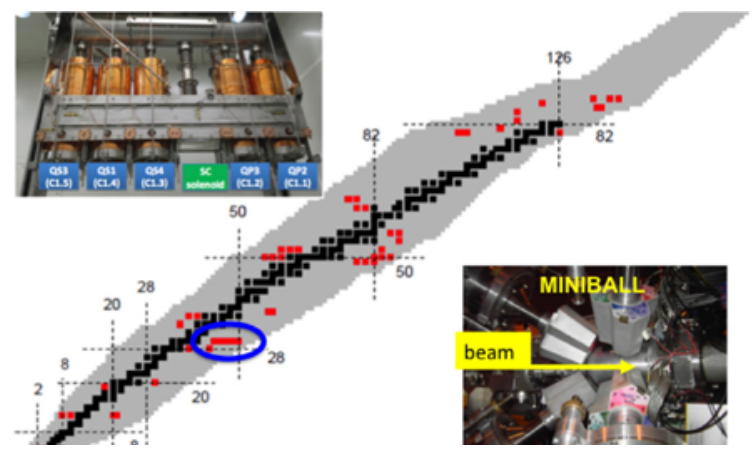

Figure 5: In the diagonal the nuclear chart shows in red the cases already approved for nuclear studies at HIE-ISOLDE. Indicated with a circle the first physics cases that will be studied this year, the n-rich $\mathrm{Zn}$ isotopes studied with Miniball. On the left a photo of the elements inside the cryo-module vessel and thermal shield are shown, the five cavities are clearly visible. On the right the Miniball setup that will be used in 2015 is shown

along the Zinc isotopic chain close to the doubly magic nucleus ${ }^{78} \mathrm{Ni}$. This study will probe recent shell-model calculations in this area of the nuclear chart. Excitation energies and connecting B(E2) values will be measured with Miniball through a multiple Coulomb excitation experiment with laser ionised purified beams of ${ }^{74-80} \mathrm{Zn}$.

\section{Summary and outlook}

The future of ISOLDE is bright. ISOLDE restarted with the low energy physics program the first of August 2014. The knowledge accumulated over decades on how to construct targets and ion sources tailored to release pure beams of specific elements are one of ISOLDE strong points. With more than 50 years since approval ISOLDE remains as the pioneer ISOL-installation both at the level of designing new devices and production of frontier physics. The first phase of HIE-ISOLDE will start for physics this autumn. The physics cases approved expand over the wide range of post-accelerated beams available at ISOLDE with more than six hundred shifts approved for physics. Our first experiment will explore the evolution of nuclear structure near ${ }^{78} \mathrm{Ni}$ by performing multi-step Coulomb excitation in n-rich $\mathrm{Zn}$ isotopes. We expect to complete the increase of energy of post accelerated beams up to $10 \mathrm{MeV} / \mathrm{u}$ in 2017.

I acknowledge the support of the ISOLDE collaboration and technical ISOLDE and HIE-ISOLDE teams. 


\section{References}

[1] E. Kugler E, Hyperfine Interactions 129 (2000) 23.

[2] E. Mané, et al., Eur. Phys. J. A42 (2009) 503.

[3] V. I. Mishin, et al., Nucl. Instrum. Methods B 73 (1993) 550.

[4] V. Fedoseyev, et al., Hyperfine Interactions, 127 (2000) 409.

[5] B. A. Marsh, et al., Nuclear Instruments and Methods in Physics Research Section B: Beam Interactions with Materials and Atoms, 317 (2013) 550.

[6] H. De Witte, et al.,Phys. Rev. Lett. 98 (2007) 112502.

[7] M. Seliverstov, et al., Eur. Phys. J., A41 (2009) 315.

[8] T. E. Cocolios, et al., Phys. Rev. Lett. 106 (2011) 052203.

[9] M. Seliverstov, et al., Phys. Rev. C 89 (2014) 034323.

[10] J. Elseviers, et al., Phys. Rev. C 84, 034307 (2011);

[11] A. N. Andreyev, et al., Phys. Rev. C 87, 054311 (2013);

[12] C. van Beveren, et al., submitted to Phys. Rev. C (2015).

[13] B. A. Marsh, et al., in preparation

[14] D. Habs, et al., Hyperfine Interactions 129 (2000) 43.

[15] D. Voulot, et al., Nucl. Instrum. Methods B 266 (2008) 4103.

[16] F Ames, et al., Nucl. Instrum. Methods A 538 (2005) 17.

[17] B Wolf, et al., Rev. Scien. Instr. 73 (2002) 682.

[18] T. Siebert, et al., Nucl. Phys. A701 (2002) 656.

[19] J Eberth, et al., Progr. Part. Nucl. Phys. 46 (2001) 389.

[20] A. Ostrowski, et al., Nucl. Instrum. Methods A 480 (2002) 448.

[21] H B Jeppesen, et al., 2005 Nucl. Phys. A 748 (2005) 374.

[22] O. Tengblad, et al., Nucl. Instrum. Methods A 525 (2004) 458. 
[23] V. Bildstein, et al., Progr. Part. Nucl. Phys. 59 (2007) 386.

[24] Th. Kröll it et al., Procedings of "Nuclear Structure and Dynamics '09 AIP Conf. Proceedings 1165 (2009) 363.

[25] P. Van Duppen and K. Riisager, J. Phys. G: Nucl. Part. Phys. 38 (2011) 024005 .

[26] L. P. Gaffney, et al., Nature 497 (2013) 199.

[27] HIE-ISOLDE, the scientific opportunities ed. By K. Riisager, P. Butler, M. Huyse and R. Krücken, CERN Report, CERN-2007-008.

[28] V. Bildstein, et al., Eur. Phys. J. A bf 48 (2012) 85.

[29] C. E. Demonchy, et al., Nuc. Instrum. Methods in Physics Research A 583 (2007) 341. 